\title{
Causas de mortalidad por colecistectomía tradicional y laparoscópica 1991-2010*
}

\author{
Drs. ATTILA CSENDES J. ${ }^{1}$, JULIO YARMUCH G. ${ }^{1}$, JUAN C. DÍAZ J. ${ }^{1}$, \\ JAIME CASTILLO K. ${ }^{1}$, FERNANDO MALUENDA G. ${ }^{1}$ \\ 1 Departamento de Cirugía Hospital Clínico de la Universidad de Chile. \\ Santiago, Chile.
}

Abstract

\section{Mortality associated with open and laparoscopic cholecystectomy}

Background: Laparoscopic cholecystectomy is nowadays the standard surgical treatment for cholelithiasis. Aim: To determine surgical mortality of laparoscopic cholecystectomy as compared with the open procedure. Material and Methods: Review of medical records of all patients subjected to open or laparoscopic surgery in a surgical service of a clinical hospital, in a period of 20 years. Results: The records of 26.441 patients were reviewed. The figures for overall, open and laparoscopic surgery mortality were $0.16,0.39$ and $0.07 \%$ respectively. Only two of the 43 deceased patients, died as a direct complication of the surgical procedure. In the rest, the causes of mortality were underlying severe medical conditions. Conclusions: Laparoscopic cholecystectomy has a very low mortality that depends mostly on underlying medical conditions and advanced age.

Key words: Cholecystectomy, cholelithiasis, mortality.

\section{Resumen}

Introducción: La colecistectomía laparoscópica se ha convertido en el gold standard de la cirugía biliar y se emplea masivamente en todo Chile. Objetivos: Determinar la mortalidad operatoria en 4 períodos de 5 años de la colecistectomía laparoscópica comparada con la colecistectomía tradicional. Material y Método: Se incluyó a todos los pacientes sometidos a colecistectomía, ya sea laparotómica o laparoscópica, entre enero de 1991 y diciembre de 2010 (20 años). Se analizó las causas de mortalidad, el grupo etario en que ocurrió y el tipo de abordaje quirúrgico. Resultados: Se operaron un total de 26.441 pacientes, con un promedio de 1.322 operados por año. La mortalidad global de la colecistectomía laparotómica fue de $0,39 \%$ y de la laparoscópica de $0,07 \%$, con un promedio general de $0,16 \%$. Las principales causas de mortalidad fueron patologías médica severas. Sólo 2 pacientes de los 43 fallecidos (5\%) tuvieron una complicación directamente derivada de la cirugía como causa de la mortalidad. Conclusiones: La colecistectomía laparoscópica es una operación de muy baja mortalidad (7 de 10.000 operados). Esta complicación se presenta principalmente en pacientes con grave patología biliar, de edad avanzada y con múltiples complicaciones médicas.

Palabras clave: Colecistectomía laparoscópica, mortalidad operatoria, colecistectomía tradicional.

*Recibido el 14 de enero de 2012 y aceptado para publicación el 12 de marzo de 2012.

Los autores no declaran conflictos de interés.

Correspondencia: Dr. Attila Csendes J.

Santos Dumont 999, Santiago, Chile.

acsendes@redclinicauchile.cl 


\section{Introducción}

La cirugía biliar benigna es la operación más frecuentemente realizada en el ámbito de la cirugía general y abdominal, desde hace muchos años en todos los servicios de cirugía del país ${ }^{1,2}$. En agosto de 1990 se introdujo en nuestro país el abordaje laparoscópico para la colecistectomía ${ }^{3}$. Rápidamente se popularizó entre los cirujanos chilenos y numerosos autores de diferentes regiones publicaron sus resultados en extenso ${ }^{4-20}$. En 1993 publicamos las estadísticas de la colecistectomía tradicional de 17 hospitales, referente a los resultados durante 1990, con énfasis en la mortalidad operatoria ${ }^{21}$. El objetivo del presente estudio fue analizar las causas de mortalidad operatoria por colecistectomía tradicional y laparoscópica, en los últimos 20 años (1991-2010) en el Departamento de Cirugía del Hospital Clínico de la Universidad de Chile.

\section{Material y Método}

\section{Pacientes estudiados}

El presente estudio es un trabajo retrospectivo, incluyendo a todos los pacientes sometidos a colecistectomía tradicional o laparoscópica entre el $1^{\circ}$ de enero de 1991 hasta el 31 de diciembre de 2010, en el Departamento de Cirugía y Emergencia del Hospital Clínico de la Universidad de Chile. Los pacientes convertidos de cirugía laparoscópica a laparotómica se consideran dentro del grupo de cirugía tradicional. Fueron excluídos los pacientes a los que se realizó coledocostomía por coledocolitiasis residual o recidivante, coledocostomía junto con la colecistectomía y a todos los pacientes con cáncer de la vesícula biliar diagnosticada en el intraoperatorio o postoperatorio por el estudio de anatomía patológica.

\section{Mortalidad operatoria}

Se la definió como el fallecimiento de un paciente hasta los 30 días del postoperado o durante la hospitalización, independiente del tiempo transcurrido desde la intervención.

\section{Cálculo estadístico}

Para la evaluación de la significación estadística, se empleó el test de Chi cuadrado, tomando un $\mathrm{p}<0,05$ como significativo.

\section{Resultados}

El total de pacientes sometidos a colecistectomía por litiasis biliar entre 1991 y 2010 fue de 26.441, con un promedio anual de 1.322 pacientes operados. La Tabla 1 muestra las cifras globales de colecistectomía tradicional y laparoscópica, realizadas en 4 períodos de tiempo de 5 años cada uno. Se aprecia que en el $1^{\mathrm{er}}$ período, cuando se desarrolló plenamente la colecistectomía laparoscópica, sólo un $45 \%$ del total de colecistectomías fue realizada por vía laparoscópica. Esta cifra fue subiendo sucesivamente hasta alcanzar un valor de $88 \%$ en el último quinquenio.

En la Tabla 2 se aprecia las cifras globales de mortalidad operatoria y las cifras de cada quinquenio, separadas según la vía de abordaje laparotómico o laparoscópico. La mortalidad global fue de $0,16 \%$, siendo $0,39 \%$ para la colecistectomía tradicional y $0,07 \%$, es decir 5 veces menos, para la colecistectomía laparoscópica $(p<0,000)$. Las cifras de mortalidad para la cirugía tradicional muestran un aumento de 8 veces comparando el $4^{\circ}$ quinquenio con el $1^{\circ}$ $(\mathrm{p}<0,000)$. Sin embargo, la cifra de mortalidad por cirugía laparoscópica se mantuvo estable en ambos quinquenios $(p>0,5 \%)$. La Tabla 3 muestra los grupos etarios de los que fallecieron, según si eran menores de 50 años, entre 51 y 69 años y sobre 70 años. Se aprecia que la mayoría de los fallecidos tenía una edad mayor a 50 años, con predominio del grupo sobre 70 años. Revisando las causas de mortalidad operatoria en pacientes bajo 50 años, que corresponde al grupo "joven", todos fallecieron por alguna causa médica que fue intratable, sin presentar complicación quirúrgica. Estos 4 fallecidos después de cirugía tradicional tuvieron como causa principal de muerte un gran absceso hepático, una malaria, una hepatitis grave fulminante y una pancreatitis aguda grave. Los 3 fallecidos después de colecistectomía laparoscópica correspondieron a una embolia pulmonar masiva, una muerte súbita por displasia del ventrículo derecho y a un accidente vascular encefálico a las $48 \mathrm{hrs}$ después de cirugía.

El grupo de 11 fallecidos entre los 51 y 69 años presentó un comportamiento similar, con una complicación quirúrgica que fue un hemoperitoneo masivo después de colecistectomía laparoscópica. Las principales causas de mortalidad después de cirugía laparotómica fueron 2 casos con pancreatitis aguda, 1 insuficiencia hepática por cirrosis, una colangitis supurada y 2 pacientes con colecistitis aguda necrótica y shock séptico. Los otros 4 fallecidos después de colecistectomía laparoscópica correspondieron a un infarto masivo del miocardio, pancreatitis aguda, hemorragia intracraneana con hidrocefalia y a una insuficiencia renal crónica en hemodiálisis.

Las causas de mortalidad en los 25 pacientes sobre 70 años fueron principalmente, colecistitis aguda necrótica en 18 pacientes con shock séptico, peritonitis biliar por permeación y absceso hepático. 
Tabla 1. Cifras globales de la colecistectomía tradicional y laparoscópica. 1991-2010

\begin{tabular}{|lccc|}
\hline Años & Total operados & $\begin{array}{c}\text { Colecistectomía } \\
\text { tradicional }\end{array}$ & $\begin{array}{c}\text { Colecistectomía } \\
\text { laparoscópica }\end{array}$ \\
\hline $1991-1995$ & 7.568 & 4.136 & $3.432(45,3 \%)$ \\
\hline $1996-2000$ & 6.561 & 1.369 & $5.192(79,1 \%)$ \\
\hline $2001-2005$ & 6.515 & 1.217 & $5.298(81,3 \%)$ \\
\hline $2006-2010$ & 5.797 & 714 & $5.083(87,7 \%)$ \\
\hline Total & 26.441 & 7.436 & 19.005 \\
\hline
\end{tabular}

Tabla 2. Cifras globales de mortalidad de la colecistectomía tradicional y laparoscópica, 1991-2010. $\mathrm{N}=\mathbf{2 6 . 4 4 1}$

\begin{tabular}{|lccccccc|}
\hline Años & \multicolumn{2}{c}{$\begin{array}{c}\text { Total } \\
\text { fallecidos } \\
\end{array}$} & \multicolumn{2}{c}{$\begin{array}{c}\text { Colecistectomía } \\
\text { tradicional }\end{array}$} & $\begin{array}{c}\text { Colecistectomía } \\
\text { laparoscópica }\end{array}$ & p \\
\hline $1991-1995$ & 10 & 0,13 & 7 & 0,17 & 3 & 0,09 & $>0,258$ \\
\hline $1996-2000$ & 3 & 0,04 & 2 & 0,14 & 1 & 0,02 & $>0,112$ \\
$2001-2005$ & 16 & 0,24 & 10 & 0,82 & 5 & 0,09 & $<0,000$ \\
$2006-2010$ & 15 & 0,25 & 10 & 1,4 & 5 & 0,1 & $<0,000$ \\
Total & 43 & 0,16 & 29 & 0,39 & 14 & 0,07 & $<0,000$ \\
\hline
\end{tabular}

Tabla 3. Grupos etarios según mortalidad por colecistectomía 1991-2010

\begin{tabular}{|c|c|c|c|c|}
\hline & \multicolumn{2}{|c|}{$\begin{array}{c}\text { Colecistectomía tradicional } \\
29 \text { fallecidos }\end{array}$} & \multicolumn{2}{|c|}{$\begin{array}{c}\text { Colecistectomía laparoscópica } \\
14 \text { fallecidos }\end{array}$} \\
\hline & $\mathbf{n}$ & Edad $x$ & $\mathbf{n}$ & Edad $x$ \\
\hline$<50$ años & 4 & 35 & 3 & 41 \\
\hline 51-69 años & 6 & 61,5 & 5 & 62,6 \\
\hline$>/=70$ años & 19 & 79,4 & 6 & 78,5 \\
\hline Menor edad & 27 & & 33 & \\
\hline Mayor edad & 94 & & 84 & \\
\hline
\end{tabular}

Tabla 4. Mortalidad global de la colecistectomía (Colecistitis crónica o aguda)

\begin{tabular}{|lccc|}
\hline & $\begin{array}{c}\mathbf{n} \\
\text { total }\end{array}$ & $\begin{array}{c}\mathbf{n} \\
\text { fallecidos }\end{array}$ & \% \\
\hline Antes 1990 (A) & 8.109 & 25 & 0,3 \\
Colecistectomía laparotómica 1991-2010 (B) & 7.436 & 29 & 0,39 \\
Colecistectomía laparoscópica (C) & 19.005 & 14 & 0,07 \\
\hline
\end{tabular}

$\operatorname{AvB} p>0,8 ; \operatorname{AvC~p~}<0,000 ; B v C p<0,000$.
Hubo además 1 paciente con mieloma múltiple, una embolia pulmonar, un infarto masivo del miocardio y 3 pacientes con cirrosis hepática e insuficiencia hepática aguda. Sólo 1 paciente falleció después de una complicación quirúrgica, que correspondió a un biliperitoneo con fístula biliar y shock séptico después de colecistectomía laparoscópica.

\section{Discusión}

Desde los inicios de la colecistectomía laparoscópica en 1990, esta técnica se desarrolló profusamente en todo Chile, desplazando en forma muy importante a la colecistectomía tradicional. En nuestro hospital, en la actualidad, el $88 \%$ de las colecistectomías, ya sean de urgencia o electivas, se realizan por vía laparoscópica. En Chile, en el último estudio publicado por nosotros ${ }^{2}$, hay hospitales públicos con una baja tasa de cirugía laparoscópica y otros con una alta tasa. Esto tiene que ver por una parte con los recursos disponibles de cada hospital y por otra parte con la experiencia del equipo quirúrgico.

El presente estudio se enfocó exclusivamente en las cifras y causas de mortalidad operatoria después de colecistectomía tradicional o laparoscópica, sin ninguna mención a la morbilidad postoperatoria. En 1993, publicamos las cifras de mortalidad operatoria en colecistectomía laparotómica en un estudio cooperativo entre 17 hospitales, referido a las cifras de mortalidad operatoria de 1990 , justo antes del comienzo de la cirugía laparoscópica, que serviría de referente a este nuevo abordaje quirúrgico. La mortalidad global de la cirugía electiva fue de $0,06 \%$ y de la colecistectomía de urgencia de $0,6 \%$, es decir, 10 veces mayor. El presente estudio confirmó una mortalidad global de $0,07 \%$ que es similar a la colecistectomía electiva por vía tradicional. En la Tabla 4 se muestran las cifras globales de la colecistectomía (sin coledocostomía), 
Tabla 5. Mortalidad de la colecistectomía en Chile (1993-2007)

\begin{tabular}{|c|c|c|c|}
\hline Autor & Año & n op. lap. & Mortalidad \% \\
\hline Hepp $^{4}$ & 1993 & $1.102^{(1)}$ & 0,09 \\
\hline Hepp $^{5}$ & 1994 & 18.718 & 0,05 \\
\hline Ibáñez ${ }^{6}$ & 1994 & 308 & 0 \\
\hline Venturelli $^{7}$ & 1994 & 613 & 0 \\
\hline Silva $^{8}$ & 1995 & $593^{(2)}$ & 0 \\
\hline Larraechea $^{9}$ & 1995 & $174^{(3)}$ & 0 \\
\hline Hepp $^{10}$ & 1996 & 35.014 & 0,04 \\
\hline Apablaza $^{11}$ & 1997 & $2.000^{(4)}$ & 0 \\
\hline Espinoza $^{12}$ & 1997 & $115 \mathrm{x}$ & 1,8 \\
\hline Fischer $^{13}$ & 1998 & $500^{(5)}$ & 0 \\
\hline Burmeister $^{14}$ & 2002 & 711 & 0 \\
\hline Cárcamo $^{15}$ & 2002 & 1.256 & 0 \\
\hline Patillo $^{16}$ & 2004 & 357 & 0 \\
\hline Adelsdorfer ${ }^{17}$ & 2004 & $799^{(6)}$ & 0 \\
\hline Acevedo $^{18}$ & 2005 & $3.254^{(7)}$ & 0,5 \\
\hline Ibáñez ${ }^{19}$ & 2007 & $5.063^{(8)}$ & 0,02 \\
\hline Montalva $^{20}$ & 2007 & $119 x$ & 0 \\
\hline MINSAL & 2007 & 22.685 & 0,07 \\
\hline
\end{tabular}

x pacientes sobre 75 años; (1) Colecistitis aguda 25\%; (2) Colecistitis aguda $100 \%$; (3) Colecistitis aguda 100\%; (4) Colecistitis aguda 15\%; (5) Colecistitis aguda $17 \%$; (6) Colecistitis aguda $37 \%$; (7) Colecistitis aguda $10 \%$; (8) Colecistitis aguda $25 \%$.

ya sea por colecistitis crónica o aguda en estos períodos diferentes. Se observa que la mortalidad global fue significativamente menor después de colecistectomía laparoscópica $(\mathrm{p}<0,000)$, comparando los diferentes períodos. Sin embargo, las cifras actuales por vía abierta son significativamente mayores (por lo menos 5 veces) que la colecistectomía laparoscópica $(\mathrm{p}<0,000)$. Este hecho tiene por lo menos 3 explicaciones, pudiendo existir otras. A.- el progresivo y explosivo aumento de la vía laparoscópica ha hecho que la experiencia de la cirugía abierta en las generaciones más jóvenes sea escasa o nula, lo que trae dificultades en el manejo quirúrgico de la cirugía abierta. Esto se ve muy claramente en el manejo actual de la coledocolitiasis, en el que se ha reemplazado la experiencia quirúrgica de la coledocostomía por el manejo endoscópico. B.- lo más probable es que en la actualidad la cirugía tradicional se reserva para casos excepcionales graves con colecistitis aguda necrótica, con absceso hepáticos, que no se resuelven por vía laparoscópica, debiendo ser convertidos a cirugía abierta. Al inicio de la experiencia en colecistectomía laparoscópica, había muchas contraindicaciones para realizarla. Con el transcurso de los años y la experiencia adquirida, prácticamente la única contraindicación es la presencia de cáncer de vesícula biliar. C.- el aumento de la longevidad de la población chilena ha producido un explosivo aumento de consultas y operaciones en pacientes sobre 75 y 80 años, quienes antiguamente se excluían completamente de un acto quirúrgico. A pesar de su uso masivo, hay un $12 \%$ de los pacientes, que justamente son los más graves y comprometidos, habitualmente de edad avanzada que terminan en una cirugía laparotómica. Estos hechos probablemente explican esta mortalidad 5 veces mayor. Las causas principales de mortalidad en los 43 pacientes fallecidos fueron de causa médica, con múltiples patologías graves y algunas poco frecuentes como malaria y mieloma múltiples. Llama la atención los 2 casos de embolia pulmonar masiva después de cirugía laparoscópica, así como las únicas 2 complicaciones quirúrgicas que causaron la muerte, que también ocurrieron después de este procedimiento. Todos los fallecidos por vía tradicional fueron principalmente por cuadro séptico secundario a colecistitis aguda necrótica. La Tabla 5 muestra las estadísticas de autores chilenos de diversas regiones que han publicado cifras de mortalidad por cirugía laparoscópica desde 1993 hasta el 2007. En esta Tabla hay 2 trabajos que se refieren exclusivamente a pacientes sobre 75 años, mostrando una mayor mortalidad en este grupo, similar a nuestros resultados. También hay algunos autores que incluyen pacientes operados de urgencia por colecistitis aguda. Al final de la Tabla se aprecia la cifra de mortalidad global el año 2007 en Chile, en 22.685 pacientes sometidos a cirugía laparoscópica tanto en hospitales públicos como clínicas privadas $^{22}$. La cifra de mortalidad es exactamente igual a la nuestra.

Las críticas al presente estudio pueden ser varias. Desde luego es una revisión retrospectiva. Los grupos no son comparables, ya que los casos más graves y complejos habitualmente terminan en cirugía laparotómica, en cambio, la vía laparoscópica es para pacientes menos complejos. La experiencia quirúrgica también es variable, ya que la vía tradicional es inversamente proporcional a la vía laparoscópica. Es decir, el aumento de la cirugía la- 
paroscópica hace decrecer la experiencia global por vía laparotómica y hace 20 años ocurría exactamente lo contrario ${ }^{23}$.

En resumen, la cirugía laparoscópica de la vesícula biliar se ha masificado y es el gold standard actual de la colecistectomía. Sin embargo, en ninguna parte corresponde al $100 \%$ de las colecistectomías, ya que hay un porcentaje bajo de pacientes que son finalmente sometidos a cirugía tradicional, ya sea de inicio o después de conversión en cirugía laparoscópica. La mortalidad actual de la colecistectomía laparoscópica es muy baja, de 7 fallecidos por 10.000 operados, principalmente en pacientes muy graves, añosos, con múltiples complicaciones médicas.

\section{Referencias}

1. Csendes A, Medina E, Medina AM. Características de los servicios de cirugía en Chile y operaciones más frecuentes realizadas en los diversos tipos de hospitales. Rev Chil Cir. 1983;111:1065-74.

2. Csendes A, González G. Cirugías digestivas más frecuentes en Chile excluyendo colorectal. Rev Chil Cir. 2008;60:379-86.

3. Lizana C. Colecistectomía por vídeo laparoscopia. 250 casos. Rev Chil Cir. 1996;43:285-91.

4. Hepp J, Ríos H, Sepúlveda R, Suárez L, Videla D, Mandujano A, et al. Colecistectomía laparoscópica: casuística del Hospital Militar. Rev Chil Cir. 1993;45:455-60.

5. Hepp J. Complicaciones de la colecistectomía laparoscópica: Encuesta nacional. Rev Chil Cir. 1994;46:56771.

6. Ibáñez L, Velasco A, López F, Zúñiga A, Guzmán S, Llanos O, et al. Colecistectomía laparoscópica, experiencia clínica . Rev Chi Cir. 1994;46:75-80.

7. Venturelli A, Jara M, Muñoz A, Díaz A, Kuschel L, Hermosilla C. Colecistectomía laparoscópica. Cuad Cir. 1994;8:46-50.

8. Silva V. Colecistectomía laparoscópica en colecistitis aguda. Rev Chil Cir. 1995;47:593-8.

9. Larrachea P, Villagrán H, Bidegain E, Fernández C, Martínez F, Ulloa O, et al. Colecistectomía laparoscópica en colecistitis aguda. Rev Chil Cir. 1995;47:371-5.

10. Hepp J, Rios H. Colecistectomía laparoscópica: evaluación de 35.014 operados en Chile. Rev Chil Cir.
1996;48:461-6.

11. Apablaza S. Colecistectomía laparoscópica. Rev Chil Cir. 1997;49:194-205.

12. Espinoza R, López F, Guzmán S, Arroyo C, Ibáñez L, Guzmán S, et al. Patología biliar en pacientes mayores de 75 años. Rev Chil Cir. 1997;49:153-6.

13. Fisher J, Irribarren O, Hernández J, Larenas E. Colecistectomía laparoscópica con o sin neumoperitoneo en colecistitis aguda. Rev Chil Cir. 1998;50:601-5.

14. Burmeister R, Apablaza S, Soto D. Coledocolitiasis en el Hospital Clínico San Borja Arriarán. Rev Chil Cir. 2002;54:148-52.

15. Cárcamo C, Venturelli A, Kuschel C, Murúa A, Díaz J, Banse C, et al. Colecistectomía laparoscópica: Experiencia del Hospital Clínico Regional Valdivia. Rev Chil Cir. 2002;54:153-8.

16. Patillo JC, Kusanovic R, Salas J, Reyes J, GarcíaHuidobro I, Sanhueza M, et al. Colecistectomía laparoscópica ambulatoria: Una experiencia factible en un hospital público chileno. Rev Med Chile 2004;132:42936.

17. Adelsdorfer W, Acevedo H, Vicuña A, Silva A. Conversión en colecistectomía laparoscópica: Experiencia Hospital San Martín de Quillota, 8 años después. Bol Hosp Viña del Mar 2004;60:16-22.

18. Acevedo A, Lombardi J, Uribe M, Catau F, Salinas C, Gallego A. Morbimortalidad y estada hospitalaria de los egresos por patología biliar del Servicio de Cirugía del Hospital del Salvador. Rev Chil Cir. 2005;412-6.

19. Ibáñez L, Escalona P, Devaud N, Montero P, Ramírez E, Pimentel F, et al. Colecistectomía laparoscópica. Experiencia de 10 años de la PUC. Rev Chil Cir. 2007;59:105.

20. Montalva S, Flisflisch H, Lena L, Cerda R, Hernández F, Matus C, et al. Resultados de la colecistectomía video laparoscópica en el adulto mayor. Rev Chil Cir. 2007;59:425-9.

21. Csendes A, Korn O, Medina E, Becerra M, Csendes P. Mortalidad de la cirugía biliar en Chile en 1990. Estudio cooperativo de 17 hospitales. Rev Méd Chile 1993;121:937-42.

22. Rajs D. Estadísticas e Informaciones Salud. MINSAL 2007.

23. Yarmuch J, Csendes A, Schutte H. Lesiones de vía biliar en 10.791 colecistectomías laparoscópicas. Rev Chil Cir. 2005;58:127-30. 